\title{
A modified sine cosine algorithm for improving wind plant energy production
}

\author{
M. H. Suid ${ }^{1}$, M. Z. Tumari ${ }^{2}$, M. A. Ahmad Ah $^{3}$ \\ ${ }^{1,3}$ University Malaysia Pahang, Malaysia \\ ${ }^{2}$ UniversitiTeknikal Malaysia Melaka, Malaysia
}

\begin{tabular}{l} 
Article Info \\
\hline Article history: \\
Received Jan 15, 2019 \\
Revised Apr 13, 2019 \\
Accepted May 8, 2019 \\
\hline Keywords: \\
Data driven \\
Modified sine cosine algorithm \\
(M-SCA) \\
Sine cosine algorithm (SCA) \\
Wind plant
\end{tabular}

Article Info

Article history

Received Jan 15, 2019

Revised Apr 13, 2019

Accepted May 8, 2019

Modified sine cosine algorithm

Sine cosine algorithm (SCA)

Wind plant

\section{Corresponding Author:}

Mohd Helmi Suid,

Faculty of Electrical and Electronics Engineering,

Universiti Malaysia Pahang,

26600 Pekan, Pahang, Malaysia.

Email:mhelmi@ump.edu.my

\begin{abstract}
This paper presents a Modified Sine Cosine Algorithm (M-SCA) to improve the controller parameter of an array of turbines such that the total energy production of wind plant is increased. The two modifications employed to the original SCA are in terms of the updated step size gain and the updated design variable. Those modifications are expected to enhance the variation of exploration and exploitation rates while avoiding the premature convergence condition. The effectiveness of the M-SCA is applied to maximize energy production of a row of ten turbines. The statistical performance analysis shows that the M-SCA provides the highest total energy production as compared to other existing methods.
\end{abstract}

Copyright $@ 2019$ Institute of Advanced Engineering and Science. All rights reserved.

\section{INTRODUCTION}

One of the primary issues of the wind plant research nowadays is that the optimal controller parameter of individual turbine does not guarantee an optimal energy production of the whole wind plant. This is due to the wake interaction between turbines in the wind plant that degrades the total energy production of the wind plant. So far, there are several of strategies that have been proposed to find the optimal controller parameters of an array of turbines in the wind plant. One of the recent popular strategies is based on the data-driven method that totally relies on the total energy production data of wind plant. These include Safe Experimentation Dynamics (SED) [1] and cooperative static control [2]. Those methods are under the class of game theoretic method that defines the controller parameter of each turbine as a player to achieve the given goal which is the total energy production. In [3], the researchers propose a Maximum energy Point Tracking (MPPT) method by incorporating a time varying energy production measurement of wind plant. Here, they show that the MPPT method provides faster convergence of total energy production than the method in [1]. Similarly, the works in [4] also considered the same model of wind plant as in [3]. Here, by manipulating the structure of the given wind plant, the proposed Multi-Resolution Simultaneous Perturbation Stochastic Approximation (MR-SPSA) is able to produce faster convergence and higher total energy production than the strategy in [3]. Other recent strategies include Bayesian Ascent [5], Random Search [6], Spiral Dynamics Algorithm [7], Particle Swarm Optimization [7] and Moth Flame Optimization [8].

On the other hand, a Sine Cosine Algorithm (SCA) [9] can also be considered as a potential tool for improving energy production of wind plant. This is because the SCA has been successfully solved various types of real world problems [10-20]. The essential features of the SCA algorithm is that the pseudo-code is 
slightly simple where the design variable is updated using only a random perturbation from the sine and cosine signals. However, based on our preliminary works using a row of ten turbines, the standard SCA is still not able to provide high accuracy of total energy production. Therefore, it motivates us to modify the standard SCA algorithm such that a better total energy production of wind plant can be achieved.

This paper presents a Modified Sine Cosine Algorithm (M-SCA) to improve energy production of wind plant. In order to solve the premature convergence issue in maximizing the energy production of wind farm, two modifications are adopted to the standard SCA as follows. Firstly, an existing linear updated mechanism in the standard SCA is modified to a new nonlinear updated mechanism, which is based on our preliminary study in [21]. Secondly, the updated design variable of each agent is modified to include an average design variable between current design variable of each agent and the best current design variable. Furthermore, a single row wind plant based on Park model [22] with ten turbines is used to assess the effectiveness of the M-SCA. The statistical performance analysis of the wind plant total energy production using the proposed method is shown. In addition, a comparative assessment between the M-SCA, the standard SCA, the PSO and the SED [1] approaches is presented.

\section{PROBLEM FRAMEWORK}

Consider the energy production of turbine $\mathrm{k}$ is defined by Ek $(\mathrm{h} 1, \mathrm{~h} 2, \ldots, \mathrm{hm})(\mathrm{k}=1,2, \ldots, \mathrm{m})$, where $\mathrm{m}$ is the total number of turbines in the wind plant and $\mathrm{hk}(\mathrm{k}=1,2, \ldots, \mathrm{m})$ is the controller parameter of each turbine k. Naturally, the incoming wind speed to a wind plant can be considered in various angle of directions with random position of turbines. Hence, the controller parameters of other turbines $\mathrm{h} 1, \mathrm{~h} 2, \ldots, \mathrm{hk}-1, \mathrm{hk}+1, \ldots$, $\mathrm{hm}$, which is not include controller parameter of turbine $\mathrm{k}$, would also influence the energy production Ek of turbine $\mathrm{k}$ due to the wake interactions between turbines. Equivalently, the changes of hk might also influence the energy productions of other turbines E1, E2, ...., Ek-1, Ek+1, ..., Em. The exact formulation of function Ek (h1, h2, ... hm) can be negligible in this study due to highly complex turbulence interactions among turbines, which are too problematic to obtain its mathematical model. Nevertheless, it can be assumed that the energy production data of each turbine is measureable. Thence, the total measured energy production can be written as follows:

$$
\bar{E}\left(h_{1}, h_{2}, \ldots, h_{m}\right)=\sum_{k=1}^{m} E_{k}\left(h_{1}, h_{2}, \ldots, h_{m}\right)
$$

Finally, this wind plant data-driven control problem can be stated as:

Problem 2.1: Find the controller parameter of each turbine $h_{k}(k=1,2, \ldots, m)$ such that the total energy production $\bar{E}\left(h_{1}, h_{2}, \ldots, h_{m}\right)$ in (1) is maximized without any knowledge on the relation between $h_{k}(k=1$, $2, \ldots, m)$ and $\bar{E}$.

\section{MODIFIED SINE COSINE ALGORITHM}

In this section, the proposed M-SCA for optimizing controller parameters of wind turbines in wind plant is explained. Firstly, a standard Sine Cosine Algorithm (SCA) is briefly described. This is followed by the description of the modification in SCA, which is able to provide better total energy production of wind plant.

\subsection{Summary of the Standard SCA}

A brief description of the standard SCA, which is introduced in [9], is shown here. Consider $g: \mathbf{R}^{n} \rightarrow \mathbf{R}$ is the cost function, vi $(\mathrm{i}=1,2, \ldots, \mathrm{N})$ is the design variable and $\mathrm{N}$ is the number of agents. Let vij $(\mathrm{j}$ $=1,2, \ldots, \mathrm{n})$ is $\mathrm{j}$-th element of the vector vi. Thence, a maximization problem is expressed as:

$$
\arg \max _{\mathbf{v}_{i}(1), \mathbf{v}_{i}(2), \ldots} g_{i}\left(\mathbf{v}_{i}(t)\right)
$$

For iteration $t=1,2, \ldots$ and for each agent $i$. The SCA algorithm updates each element of design variable for each agent iteratively as:

$$
v_{i j}(t+1)= \begin{cases}v_{i j}(t)+r_{1} \sin \left(r_{2}\right) \times\left\|r_{3} P_{j}-v_{i j}(t)\right\| & \text { if } r_{4}<0.5, \\ v_{i j}(t)+r_{1} \cos \left(r_{2}\right) \times\left\|r_{3} P_{j}-v_{i j}(t)\right\| & \text { if } \quad r_{4} \geq 0.5,\end{cases}
$$


Where,

$$
r_{1}=a-\frac{a t}{T}
$$

for maximum iteration $\mathrm{T}$ and constant positive value a. Note that $\mathrm{r} 2, \mathrm{r} 3$ and $\mathrm{r} 4$ are random values that are generated independently and uniformly in the ranges $[0,2 \pi],[0,2]$ and $[0,1]$, respectively. The detailed justification on the selection of the coefficients $r 1, r 2, r 3$ and $r 4$ are clearly explained in [9]. In (3), the symbol $\operatorname{Pj}(\mathrm{j}=1,2, \ldots, n)$ is denoted as the best current design variable in $\mathrm{j}$-th element that is kept during tuning process. Please see [9] for the detail of the SCA pseudo-code.

\subsection{Modified SCA (M-SCA)}

There are two modifications are proposed to the original SCA, which are the updated step size $\mathrm{r} 1$ in (4) and the updated in (3). In the original version of SCA, the $\mathrm{r} 1$ value is linearly decayed from a to 0 during the tuning process, which is claimed to provide an exact balance between exploration and exploitation. Nevertheless, the setting in (4) maybe limited to several applications only, while it is good to propose more generic of $\mathrm{r} 1$ that can cover more general class of applications. Motivated from the above limitation, (4) is modified to produce a new generic of updated step size as follows:

$$
\hat{r}_{1}=a\left(1-\left(\frac{t}{T}\right)^{a}\right)^{g}
$$

which is directly adopted from our previous work in [21]. In (5), the symbols $\alpha$ and $\gamma$ are the positive constant values that are introduced to regulate the portion of exploration and exploitation during the tuning process. Although there are several researchers that proposed exponential [23] and quadratic [24] curves in the original $\mathrm{r}$, their are limited to only one curve, while (5) can generate two curves during the whole iterations. As a result, it is expected that our new ${ }^{\hat{r}_{1}}$ can provide more choices of exploration and exploitation portions as compared to the exponential and quadratic versions.

On the other hand, the original version of SCA updates each element of the design variable by randomly perturb the current vij using the sine or cosine function that is multiplied with absolute error of the best current design variable and current design variable of each agent as shown in (3). However, there is high possibility of the current updated design variable vij(t+1) in (3) traps in local minima since the existing random perturbation alone is still not enough to avoid this premature convergence. In order to solve this issue, it is better to perturb an average element of both vij(t) and $\mathrm{Pj}$, instead of perturbing only the current vij(t) in (3). As a result, the current best design parameter can assist any outliers design variable to jump out from the local minima and continues a new searching track. Therefore, the new updated is proposed as follows:

$$
v_{i j}(t+1)=\left\{\begin{array}{l}
\frac{v_{i j}(t)+P_{j}}{2}+r_{1} \sin \left(r_{2}\right) \times\left\|r_{3} P_{j}-v_{i j}(t)\right\| \text { if } r_{4}<0.5, \\
\frac{v_{i j}(t)+P_{j}}{2}+r_{1} \cos \left(r_{2}\right) \times\left\|r_{3} P_{j}-v_{i j}(t)\right\| \text { if } r_{4} \geq 0.5 .
\end{array}\right.
$$

Finally, the M-SCA will follow the same SCA by replacing (3) and (4) with (6) and (5), respectively.

\subsection{Applications of M-SCA for Improving Energy Production of Wind Farm}

The procedure for data-driven control of wind plant based on M-SCA is given as follows:

Step 1: Select the values of $\alpha$ and $\gamma$ in (5).

Step 2: Execute the M-SCA by setting $\bar{E}=g_{i}$ and $h_{k}(k=1,2, \ldots, m)=v_{i j}(j=1,2, \ldots, n)$ for each $i$.

Step 3: The algorithm stops with the solution $h_{k}{ }^{*}(k=1,2, \ldots, m)=P_{j}(j=1,2, \ldots, n)$ after $T$ iterations and the corresponding total energy production $\bar{E}\left(h_{1} *, h_{2} *, \ldots, h_{m}{ }^{*}\right)$ is observed.

The detail of the pseudo-code can be downloaded from the following link: http://bit.do/m-sca. 


\section{NUMERICAL RESULTS}

The effectiveness of the M-SCA is observed in this section. The performance of M-SCA is verified on a 10 turbines row wind plant as presented. The wind plant model based on the Park model [22] is adopted for ten turbines $(\mathrm{m}=10)$ with $80 \mathrm{~m}$ of turbine rotor diameter on each. Please see [22] for the detail of the wind plant model. The location of wind turbine is positioned in a row with a same $560 \mathrm{~m}$ distance between each turbine. The values of incoming wind speed, air density and the turbulence gradient parameters are $\mathrm{U}=$ $8 \mathrm{~m} / \mathrm{s}, \rho=1.225 \mathrm{~kg} / \mathrm{m} 3$ and $\varphi=0.04$, respectively.

In this work, a comparative assessment between the proposed M-SCA and the original SCA, PSO and SED in terms of maximum total energy production is considered. The coefficients of M-SCA is set as $\alpha$ $=0.01, \gamma=1$ and $\mathrm{a}=0.43$, with $\mathrm{N}=10$ and $\mathrm{T}=1000$, after performed several initial investigations. Similarly, the coefficient of SCA is set as a $=2$, with the same combinations of $\mathrm{N}$ and $\mathrm{T}$ as M-SCA. The coefficients of PSO is fixed as $\mathrm{c} 0=0.9, \mathrm{c} 1=0.1$, and $\mathrm{c} 2=0.5$, with $\mathrm{N}=20$ and $\mathrm{T}=500$, to produce the same number of function evaluations. Please see [25] for the details of the PSO algorithm. Meanwhile, the SED with interval step size $K G=0.03$, the probability of changing the design variable $\mathrm{e}=0.3$ and $\mathrm{T}=10,000$ are adopted. Please see [1] for the detail of SED algorithm. The initial control parameter of each turbine for M-SCA, SCA and PSO are set randomly between ranges of $[0,1 \backslash 3]$, while the initial control parameter of SED is set at $1 / 3$ for all turbines.

Table 1 tabulates the statistical performance of the total energy production after 100 independent trials in terms of its mean, best, worst and standard deviations. It can be seen that the M-SCA yields the highest average total energy production, followed by the PSO, SED and the SCA. A similar trend can be observed for the corresponding best and worst values. Furthermore, the proposed M-SCA also produces slightly lower value of standard deviation than the SCA, PSO and SED. The finding justifies that the proposed M-SCA is robust to the stochastic effect while consistently improving total energy production. On the other hand, the best optimal controller parameters of the M-SCA are recorded as hk $(\mathrm{k}=1,2, \ldots, 10)=$ $(0.2061,0.1611,0.1648,0.1651,0.1698,0.1173,0.2258,0.1877,0.1837,0.3333)$. It shows that the optimum values of the controller parameters of the upstream wind turbines are lower than the $1 / 3$ to reduce the turbulence effect and increase the accumulation wind speed to the downstream turbines. In contrast, since there is no more downstream turbine for the final turbine, its controller parameter is fixed at the full capacity of $1 / 3$. This trend is similar to existing investigation on data-driven control of wind plant, e.g., [1], while improving the total energy production.

Table 1. Comparative Assessment of Total Energy Production Between M-SCA, SCA, PSO and SED

\begin{tabular}{ccccc}
\hline Statistical results & M-SCA & SCA [9] & PSO [25] & SED [1] \\
\hline Mean (MW) & 4.7648415722 & 4.6337747727 & 4.7648415625 & 4.7644075485 \\
Best (MW) & 4.7648415723 & 4.7380950026 & 4.7648415723 & 4.7648415242 \\
Worst (MW) & 4.7648415719 & 4.4327003068 & 4.7648414855 & 4.7627457259 \\
Standard Deviation & 0.00010657 & 83861.0326 & 0.0141665007 & $4.513106 \times 10^{2}$ \\
\hline
\end{tabular}

\section{CONCLUSION}

In this study, a new Modified Sine Cosine Algorithm (M-SCA) for improving wind farm control parameters has been presented. The proposed M-SCA is verified on a single row wind plant of 10 turbines. The results prove that the two proposed modifications on the original SCA have significantly improved the total energy production of wind plant. In particular, the M-SCA has produced a slightly higher total energy production than the original SCA, PSO and SED with more consistent results. Thence, it justifies the potential of M-SCA for data-driven control of wind plant.

\section{ACKNOWLEDGEMENTS}

The work was fully supported by University Malaysia Pahang under the MOE Fundamental Research Grant FRGS/1/2017/ICT02/UMP/02/2 or RDU170104.

\section{REFERENCES}

[1] Marden JR, Ruben SD, Pao LY,"A model-free approach to wind farm control using game theoretic methods", 2013 IEEE Transactions on Control Systems Technology, 21p 1207

[2] Park J, Kwon S and Law KH 2013, "Wind farm power maximization based on a cooperative static game approach", Proc. SPIE 8688.

[3] Gebraad PM and van Wingerden JW, "Maximum power-point tracking control for wind farms", 2015 Wind 
Energy, 18 pp. 429.

[4] Ahmad MA, Azuma SI and Sugie T, "A model-free approach for maximizing power production of wind farm using multi-resolution simultaneous perturbation stochastic approximation" 2014 Energies, 7 pp. 5624.

[5] Park J and Law KH, "A data-driven, cooperative wind farm control to maximize the total power production", 2016 Applied Energy, 165 pp. 151.

[6] Ahmad MA, Hao MR, Raja Ismail RMT, Nasir ANK 2016, "Model-free wind farm control based on random search", Proc. of IEEE International Conference on Automatic Control and Intelligent Systems, pp. 131.

[7] Hao MR, Raja Ismail RMT, Ahmad MA 2017, "Using Spiral Dynamic Algorithm for Maximizing Power Production of Wind Farm", Proc. of IEEE International Conference on Applied System Innovation, pp. 1706.

[8] Md Idris MA, Hao MR, Ahmad MA,"A Data Driven Approach to Wind Plant Control using Moth-Flame Optimization (MFO) Algorithm”, 2019 International Journal on Advanced Science, Engineering and Information Technology, 9(1), pp. 18-23.

[9] Mirjalili S, "SCA: a sine cosine algorithm for solving optimization problems", 2016 Knowledge-Based Systems, 96 pp. 120.

[10] Sindhu R, Ngadiran R, Yacob YM, Zahri NAH and Hariharan, "M Sine-cosine algorithm for feature selection with elitism strategy and new updating mechanism”, 2017 Neural Computing and Applications, 28 pp. 2947.

[11] Das S, Bhattarcharya A, Chakraborty AK, "Solution of short-term hydrothermal scheduling using sine cosine algorithm”, 2018 Soft Computing, 22 pp. 6409.

[12] Reddy KS, Panwar LK, Panigrahi BK and Kumar R. "A New Binary Variant of Sine-Cosine Algorithm: Development and Application to Solve Profit-Based Unit Commitment Problem", 2018 Arabian Journal for Science and Engineering, 43 pp. 4041.

[13] Wang J, Yang W, Du P, Niu T, "A novel hybrid forecasting system of wind speed based on a newly developed multi-objective sine cosine algorithm”, 2018 Energy Conversion and Management, 163 pp. 134-150.

[14] Li S, Fang H, Liu X, "Parameter optimization of support vector regression based on sine cosine algorithm", 2018. Expert Systems with Applications, 91 pp. 63-77.

[15] Attia AF, El Sehiemy RA, Hasanien HM, "Optimal power flow solution in power systems using a novel SineCosine algorithm", 2018 International Journal of Electrical Power \& Energy Systems, 99 pp. 331-343.

[16] Nenavath, H. and Jatoth, R.K., 2018. "Hybridizing sine cosine algorithm with differential evolution for global optimization and object tracking". Applied Soft Computing, 62, pp.1019-1043.

[17] Gupta S, Deep K, "Improved sine cosine algorithm with crossover scheme for global optimization", 2019 Knowledge-Based Systems, 165 pp. 374-406.

[18] Mahdad B, Srairi K, "A new interactive sine cosine algorithm for loading margin stability improvement under contingency”, 2018 Electrical Engineering, 100(2) pp. 913-933.

[19] Rizk-Allah RM, "Hybridizing sine cosine algorithm with multi-orthogonal search strategy for engineering design problems", 2018 Journal of Computational Design and Engineering, 5(2) pp. 249-273.

[20] Issa M, Hassanien AE, Oliva D, Helmi A, Ziedan I, Alzohairy, "A ASCA-PSO: Adaptive sine cosine optimization algorithm integrated with particle swarm for pairwise local sequence alignment", 2018 Expert Systems with Applications, 99 pp. 56-70.

[21] Suid MH, Ahmad MA, Raja Ismail RMT, Ghazali MR, Irawan A, MohdTumari MZ 2018, “An improved sine cosine algorithm for solving optimization problems", Proc. of IEEE Conference on Systems, Process and Control to appear.

[22] Grunnet JD, Soltani M, Knudsen T, Kragelund M and Bak T 2010, "Aeolus toolbox for dynamic wind farm model, simulation and control", Proc. Eur. Wind Energy Conference, pp. 3119.

[23] Qu C, Zeng Z, Dai J, Yi Z, He W, “A Modified Sine-Cosine Algorithm Based on Neighborhood Search and Greedy Levy Mutation”, 2018 Computational intelligence and neuroscience, pp. 1.

[24] Yong L and Liang M, "Sine cosine algorithm with nonlinear decreasing conversion parameter", 2017 Computer Engineering and Applications, 53 pp. 1.

[25] Kennedy J and Eberhart RC, "Particle swarm optimization 1995", Proc. IEEE Int. Conference on Neural Networks, pp. 1942.

\section{BIOGRAPHIES OF AUTHORS}

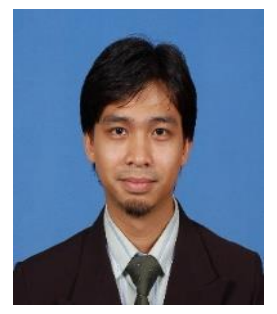

Mohd Helmi Suid received the B.Eng (Hons.) in Electrical and Electronics Engineering from Universiti Teknikal Melaka in 2007. In 2014, he receives M.Sc from School of Electronic and Electrical Engineering, University Sains Malaysia, Penang. He is currently, a lecturer in Faculty of Electrical and Electronics Engineering, University Malaysia Pahang (UMP). His current research interests are digital image processing, vision for robotics application and computational intelligence. 


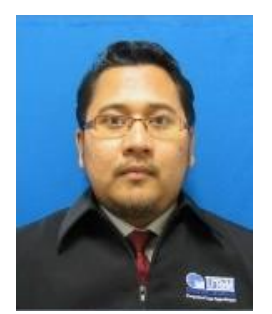

Mohd Zaidi Mohd Tumari received his first degree in B.Eng. Electrical Mechatronics in 2008 from Universiti Teknologi Malaysia (UTM) in Johor, Malaysia. In 2010, he received a Master degree in M.Eng. Mechatronics and Automatic Control from Universiti Teknologi Malaysia (UTM). He has an experience as a research assistant at Robotic Laboratory in UniversitiTeknologi Malaysia (UTM) in 2008, Tutor in Universiti Pendidikan Sultan Idris (2008-2011) and Lecturer in Universiti Malaysia Pahang (2011-2014). Currently, he is a Lecturer in Faculty of Engineering Technology, Universiti Teknikal Malaysia Melaka (UTeM).As an academician and researcher, he has involved in many research and development in field of mechatronics, control and robotics. He has currently published more than 31 paper works in various International Conference and Journals in the field of mechatronics, control and robotics. He also became a professional member in MBOT, BEM and IEM. His current research interests are optimization, vibration and sway control, liquid slosh system and vehicle steer by wire system.

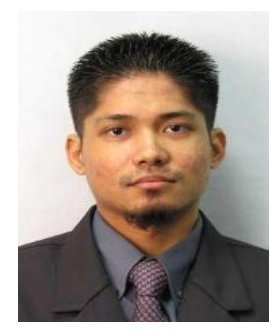

Mohd Ashraf Ahmad received his first degree in B.Eng Electrical Mechatronics and Master Degree in M.Eng Mechatronics and Automatic Control from University of Technology Malaysia (UTM) in 2006 and 2008, respectively. In 2015, he received a Ph.D in Informatics (Systems Science) from Kyoto University. Currently, he is a lecturer in Faculty of Electrical and Electronics Engineering, University Malaysia Pahang (UMP). His current research interests are model-free control, control of mechatronic systems, nonlinear system identification and vibration control. 